\title{
DECISION-CTO: A "negative" clinical trial? Really?
}

\begin{abstract}
Last March the results of the DECISION-CTO randomised trial were finally presented during the ACC Congress in Washington. This is a long-awaited paramount piece of evidence, because it is the largest randomised clinical trial comparing a strategy of percutaneous revascu-
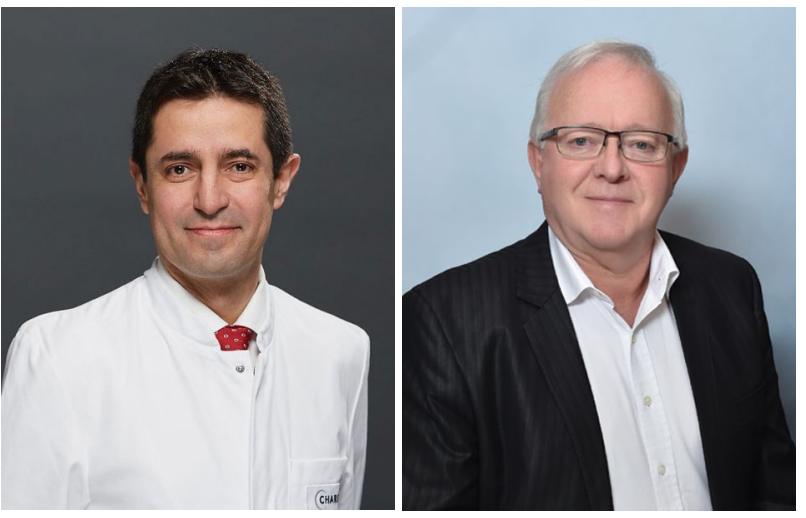
larisation in chronic total occlusions (CTO) vs. optimal medical treatment (OMT) hitherto. The trial however failed to show any significant difference in the primary composite endpoint (mortality of any cause, non-fatal myocardial infarction [MI] and any revascularisation) at 3-years follow-up, so it has been presented as a "negative" trial for percutaneous coronary intervention (PCI) in this challenging scenario, or in a more positive formulation, as "non-inferiority" of OMT vs. PCI.

It is somewhat surprising the general defeatism that has pervaded the interventional community after these apparently disappointing results, even among CTO-experts. Uncountable press-releases highlight a discouraging message, with striking words like "negative", "fails", "nonsignificant", etc. Key opinion leaders surrendered to the general panic and tried to find refuge in polyvalent commonplaces, like the need to select better the patients, the indisputable benefit of an interventional approach in some cases after their personal experience, or the geographical frame of the study, that rather sounded like an attempt to save some personal belongings from the anticipated tsunami. These remarks implicitly accept and convey a negative result from the trial. Only my dear friend Davide Capodanno in his PCR news was cautious enough as to interpret the trial as negative, but concluding that the result leaves the question about the benefits of PCI in CTOs essentially unanswered.

Is DECISION-CTO a negative trial indeed? Formally it is so, no question, because it has failed to prove superiority in the predefined primary endpoint. For those who like to interpret the clinical trials in simplistic soccer terms, this will ever remain the devastating summary of such a precious collection of data: PCI 1 - OMT 1 , tie, therefore the safest and cheapest option, namely OMT, prevails. This might be a good approach for soccer, maybe also for clinical routine, since we are often confronted with decisions that appear as a sharp dichotomy without nuances. Nonetheless, before accepting the defeat of PCI, it might be worth analysing the data with some deeper detail.

- The estimated sample size was 1284 , however the trial was prematurely stopped due to difficulties to enrol patients. The final number of patients included was 834. Moreover, most of patients came from a single centre, very active in the recruitment.

- The cross-over was not negligible: 72 patients in the OMT arm (18.1\%) underwent PCI, whilst 65 patients in the PCI arm (15.6\%) were treated with OMT (36 of them due to failed PCI).

- Peri-procedural MI, defined as an increase x5 in the levels of cardiac markers, was included as part of the primary endpoint. Actually the differences in non-fatal MI were exclusively due to the differences in periprocedural MI, but there were no differences in spontaneous MI along the reported 5 years follow-up.

- PCI was penalised with more non-fatal MIs (namely periprocedural) and revascularisations, nonetheless the overall mortality in the PCI arm was sensibly lower than in the OMT arm (3.0\% vs. $4.4 \%$ at $3 y$ follow-up, and $4.5 \%$ vs. $7.9 \%$ at $5 y$ follow-up), with a reduction of $50 \%$ in the particular case of cardiac death at the $3^{\text {rd }}$ year ( $1.9 \%$ vs. $\left.3.6 \%\right)$. Of course these differences are not significant, but the reported 
number at risk is as low as 409 at 3 years and just 155 at the $5^{\text {th }}$ year. This means that the follow-up is still incomplete in many cases or that the study was undermined by unacceptable loss at follow-up.

- The incidence of stroke was 5-fold higher in the OMT group (5\% vs. $1 \%$ ) at 5 years, close to reach statistical significance, although the patients at risk at that point were only 151 .

- In the per-protocol and as-treated analysis, PCI was superior to OMT, so the latter failed to prove non-inferiority. The superiority of PCI for the composite endpoint was close to reach statistical significance, even though the patients at risk were only 353 at the $3^{\text {rd }}$ year of follow up and 137 at the $5^{\text {th }}$ year.

In light of the above, I perceive DECISION-CTO as an utmost encouraging trial, close to prove the clinical advantages of an interventional strategy in patients with a CTO. I see no "negative trial" at all, but maybe this is just because I am not so much into soccer.

As statistician I see just an underpowered trial with problems in its design and execution. It was clearly a mistake in the design including the peri-procedural MI, because it burdens considerably the PCI arm. Such complex interventions are almost systematically associated with an increase in cardiac markers due to the technique (occlusion of small side branches, subintimal courses, stenting of long segments, transient occlusion of the collaterals, reversal of the collateral flow, etc.), in most of cases without any clinical consequence at all. The highest increases in cardiac markers happen in the CTOs with the largest myocardium in jeopardy, corresponding paradoxically to the patients who benefit the most from the intervention. Most interestingly, the data obtained allow us to calculate quite precisely the required sample size to demonstrate a net benefit in a major endpoint like mortality.

- If the primary endpoint had been all-cause mortality at 5 years, in the intention-to-treat analysis, a sample size of 1696 patients would have resulted in a statistically significant difference. The completion of the initially-planned sample size would still remain non-significant, but just an increase in $32 \%$ would have rendered a statistically significant difference: PCI 1 - OMT 0, for our soccer fans. In that scenario, it is important to notice that the number-needed-to-treat is just 29 patients, i.e. we need to perform just 29 CTO-PCIs to spare one live at 5 years follow-up.
- The cross-over introduced too much noise as to draw a too assertive conclusion. Actually in the as-treated analysis, OMT failed to appear as non-inferior to PCI, because PCI performed clearly better in the composite endpoint. If there had been no cross-over, a sample size of 1282 would have resulted in a statistically significant difference favouring PCI. Actually this is quite precisely the estimated sample size of the investigators, that must be congratulated for their excellent job. It was just the cross-over, impossible to anticipate in such complex trials, the factor that somehow ruined their accurate calculation. In this scenario, the number-needed-to-treat (NNT) is just 15 patients, i.e. we need to treat SUCCESFULLY just 15 CTOs to spare one life, MI, stroke or to avoid a revascularisation at 5 years follow-up. Similar results, for the same endpoint at 3 years follow-up, with a NNT $=18$ patients.

- The differences in stroke will become statistically significant when the follow-up is completed in all patients, if the current trend is maintained (only 670 are required for that), favouring the PCI.

As interventional cardiologist, the trial offers lots of fascinating pearls that confirm or challenge my current perception about CTO-PCI. I am not truly surprised for the benefit in mortality, because it confirms somehow the data reported in previous observational or post-hoc studies [1-6]. I find also clinically unacceptable stating that a treatment is non-inferior to another one, based on a composite endpoint, when its different components move clearly in different directions for each therapeutic option. This is acceptable if all the components of the endpoint move consistently in the same direction, but it makes absolutely no sense trading off lives or strokes (where PCI performs better) for troponins and recaths (where OMT performs better). As physician or as patient I would not hesitate. Furthermore, OMT is clearly inferior to PCI in the per-protocol or in the as-treated analysis, very close to reach statistical significance, as we have already discussed. Therefore, we just need to offer our patients a successful revascularisation. We do not need to select the patients better, we need to select the operators better.

I find also very interesting noticing that the success rate was as high as $91.1 \%$ and that retrograde approaches were used in $24.6 \%$ of cases, because this speaks in favour of the quality of the 
operators and of the interventions performed, corresponding to a modern, professional and dedicated CTO-program. Still the use of IVUS was only $6 \%$, even though it can be instrumental in complex CTO-interventions.

Finally, some data are intriguing. Excitingly intriguing is the observation that the subgroup which seems to benefit the most from the PCI is that of the patients without angina. This challenges definitely the common established perception. Disappointingly intriguing is the observation that PCI does not improve the quality of life or the frequency of angina, because it is at variance with my daily experience treating patients with CTOs. These data will require further elaboration and need to be confirmed in future studies.

In summary, perhaps DECISION-CTO might have failed to reach its predefined endpoints, but has provided us with the information necessary to prove the clinical benefit of $\mathrm{PCI}$ in CTO in a future trial. Therefore, I perceive DECISION-CTO as a positive and encouraging trial: the investigators must be congratulated for an excellent job. They have taught us a lot of lessons that must be learned in the planning of subsequent studies. We cannot underestimate the logistic problems, like the limited number of operators able to warrant an acceptable success rate, the difficulties to recruit and randomise patients, the unavoidable cross-over, etc. Still we must find the way to surmount all these limitations, because soccer is most popular in our society, including cardiologist, so it is mandatory to design a match in which PCI can win. Otherwise we will not convince our sceptical soccer-fan col- leges, no matter how thoughtful considerations we present them.

\section{Conflict of interest: None declared}

\section{References}

1. Mehran R, Claessen B, Godino C, et al. Long-Term outcome of percutaneous coronary intervention for chronic total occlusions. JACC Cardiovasc Interv. 2011; 4(9): 952-961, doi: 10.1016/j. jcin.2011.03.021.

2. Claessen BE, Dangas GD, Weisz G, et al. Prognostic impact of a chronic total occlusion in a non-infarct-related artery in patients with ST-segment elevation myocardial infarction: 3-year results from the HORIZONS-AMI trial. Eur Heart J. 2012; 33(6): 768775, doi: 10.1093/eurheartj/ehr471, indexed in Pubmed: 22240495.

3. Rosner GF, Kirtane AJ, Genereux P, et al. Impact of the presence and extent of incomplete angiographic revascularization after percutaneous coronary intervention in acute coronary syndromes: the Acute Catheterization and Urgent Intervention Triage Strategy (ACUITY) trial. Circulation. 2012; 125(21): 2613-2620, doi: 10.1161/CIRCULATIONAHA.111.069237, indexed in Pubmed: 22550156.

4. George S, Cockburn J, Clayton T, et al. Long-Term follow-up of elective chronic total coronary occlusion angioplasty: analysis from the U.K. Central Cardiac Audit Database. J Am Coll Cardiol. 2014; 64(3): 235-243, doi: 10.1016/j.jacc.2014.04.040.

5. Ladwiniec A, Allgar V, Thackray S, et al. Medical therapy, percutaneous coronary intervention and prognosis in patients with chronic total occlusions. Heart. 2015; 101(23): 1907-1914, doi: 10.1136/ heartjnl-2015-308181, indexed in Pubmed: 26552758.

6. Farooq V, Serruys PW, Garcia-Garcia HM, et al. The negative impact of incomplete angiographic revascularization on clinical outcomes and its association with total occlusions: the SYNTAX (Synergy Between Percutaneous Coronary Intervention with Taxus and Cardiac Surgery) trial. J Am Coll Cardiol. 2013; 61(3): 282-294, doi: 10.1016/j.jacc.2012.10.017, indexed in Pubmed: 23265332.

\author{
Juan Luis Gutiérrez-Chico ${ }^{1}$, MD, PhD, FESC, FACC, Yves Louvard ${ }^{2}$, MD, FSCAI \\ ${ }^{1}$ DRK-Klinikum Westend, Berlin, Germany \\ ${ }^{2}$ Institut cardiovasculaire Paris-Sud (ICPS), \\ Ramsay Générale de Santé, Massy, Quincy, France
}

